\title{
Errors in auditory direction-finding after compensation for visual re-arrangement '
}

JEROLD H. REKOSH AHD SAHFORD J. FREEDMAN

TUFTS UNIVERSITY

Ss walked about out of doors wearing laterally displacing prisms, and sound-attenuating muffs. Errors occurred in an auditory localization task during exposure to visual displacement. With continued exposure these errors tended to disappear after about $180 \mathrm{~min}$. The errors disappeared earlier when muffs were not worn.

The past 10 years has seen the development and application of a number of techniques in experimental psychology loosely referred to as sensorimotor rearrangement. (Smith and Smith reviewed the older literature in 1962, and Wohlwill the more recent studies in 1966). These procedures perturb the accustomed relationships between man and his perceptual environment in order to study his sensorimotor plasticity and adaptive limits. The test procedures employed invariably require precise, spatially-oriented behavior. Although such experiments have not been explicitly considered in this light, their effect is to disrupt the normal congruence between and among different spatial senses. For example, shifting the optical array to the right or to the left results in a soundstimulating object being heard and seen in two different places simultaneously. However, most Es have measured effects only in terms of the modality that the experimental condition had been designed to perturb neglecting the fact that what has been perturbed is a set of relationships.

There is evidence that the effects of compensation for optical displacement are not limited to vision. After partial compensation in visually guided reaching, Harris (1963) found that Ss pointed incorrectly both at auditory targets and at "straight ahead." Hay and Pick (1966) in a long-term study reported similar effects; with prolonged exposure the errors in auditory pointing and pointing "straight ahead" diminished. Freedman and Gardos (1965) have described changes in visually guided reaching accompanying compensation for auditory re-arrangement. (Craske, in 1966, also described changes in visually guided arm movements following adaptation to auditory displacement; the experimental method and type of movement were radically different however.)

The present experiments were designed specifically to test for intersensory effects, the hypothesis being that changes in auditory localization would be associated with compensation for visual re-arrangement.

\section{Procedure}

Two experiments were performed employing a total of 28 male college student $\mathrm{Ss}$ recruited by the student employment office.
Experiment $1(N=12)$. Ss walked about out of doors for $45 \mathrm{~min}$ wearing monocular laterally displacing prism goggles (16.4 diop. base right or base left) and sound attenuating muffs. Before and after exposure, visual and auditory direction-finding were tested: $S$ manipulated the position of a stimulus at a distance of $3 \mathrm{ft}$ from the center of his head until he judged it to be directly in front of him. As stimuli, 10 settings of a small pulsed "white" noise were followed by 10 settings of a $1 \mathrm{in}$. $1 / 16 \mathrm{in.}$ luminous vertical line. Differences between the means of the 10 pre- and postexposure settings for each session were taken as measures of shifts in auditory and visual directionfinding.

Tests were conducted without either prisms or muffs in a dark, quiet room. The $s$ used his right eye for exposure and for testing; the left eye was occluded. Head motion was restricted by a bite board. Each $S$ participated in two control sessions followed by four experimental sessions. In the control sessions, which were balanced for starting position of the test stimuli, S walked about for $45 \mathrm{~min}$ wearing goggle frames without lenses and sound-attenuating ear muffs. His left eye was covered. The four experimental sessions were arranged according to a Latin Square design balanced for prism base (left and right) and starting position of test stimuli (left and right).

Experiment $2(N=16)$. Ss walked about out of doors for $4 \mathrm{~h}$ wearing monocular prism goggles. Five auditory followed by five visual midline judgments were made every $30 \mathrm{~min}$ using the same procedure as in Experiment 1. The first eight Ss wore sound-attenuating muffs as in Experiment 1; the second eight wore no muffs. Each $\mathrm{S}$ particlpated in one control session and one experimental session; each was assigned one prism base for exposure and one starting position of stimuli for testing in a balanced design.

\section{Results}

Experiment 1. Table 1 summarizes the findings in the first experiment. Compensatory shifts in setting

Table 1. Mean Shifts in Median-Plane Judgments After Active Whole-Body Exposure to Displaced Vision ( $N=12$ )

\begin{tabular}{clrr} 
Runs/Subject & \multicolumn{1}{c}{ Condition } & Audition & Vision \\
\hline 2 & Base Right & $-3.17^{\circ}$ & $-2.25^{\circ}$ \\
2 & Base Left & $+2.60^{\circ}$ & $+3.18^{\circ}$ \\
4 & Total Experimental & $2.88^{\circ}$ & $2.72^{\circ}$ \\
2 & Control (No Prism) & $+0.25^{\circ}$ & $+0.06^{\circ}$ \\
\hline
\end{tabular}

Note: All experimental shifts significant in predicted direction $p<$ .005 (Wilcoxon). 


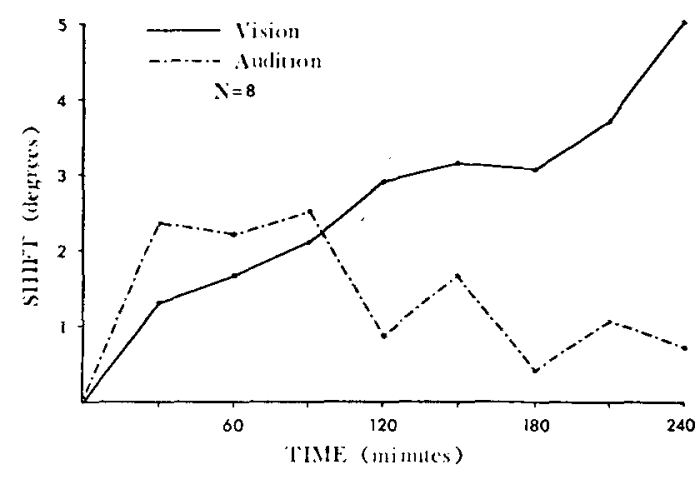

Fig. 1. Mean shifts in visual and auditory median-plane judgments during prolonged exposure to displaced vision: attenuated auditory stimulation.

visual targets in the apparent median plane were accompanied by. equivalent auditory shifts in the same direction: mean visual shift, $2.72^{\circ}$; mean auditory shift, $2.88^{\circ}$. All experimental shifts were significant in the predicted direction, and of the 96 mean shifts calculated, 46 of 48 were in the predicted direction for vision and 47 of 48 were in the same direction for audition. An analysis of variance of these data indicated that the independent variables of prism base, starting position of test stimuli, and sense modality did not significantly contribute to the main effects.

Experiment 2. Figure 1 summarizes the experimental findings for the first group of eight $4-\mathrm{h}$ Ss. Both visual compensatory shifts and auditory maladaptive shifts were about $2^{\circ}$ and not significantly different from one another for the first $90 \mathrm{~min}$. After that the mean auditory shift decreased, and at $120 \mathrm{~min}$ was significantly less than the visual ( $p<.01$, Wilcoxon). The two continued to separate to the end of the experiment where the mean visual shift was $5.0^{\circ}$ and the mean auditory shift was less than $1 . \theta^{\circ}$ (visual-auditory difference ( $\mathrm{p}<.005$, Wilcoxon).

Figure 2 shows that for the eight $4-\mathrm{h}$ Ss who did not wear ear muffs, a mean significant maladaptive auditory shift was measured only at $30 \mathrm{~min}$; this was significantly less than the compensatory visual shift at $30 \mathrm{~min}(\mathrm{p}<.005$, Wilcoxon). Also, the rate of visual compensation was much faster with unimpeded auditory stimulation. None of the means of the later auditory measurements were significantly different from zero. The compensatory visual shift seemed to plateau at about $4^{\circ}$ and remain at that level to the end of the experiment.

The eight 4-h Ss who wore sound-attenuating muffs manifested no significant difference between the means for pre- and postexposure tests in either vision or audition under a control condition (walking about wearing monocular empty goggle frames). The mean visual shift was $0.3^{\circ}$ to the left; the mean auditory shift, $0.8^{\circ}$ to the right. The eight Ss who did not wear muffs also manifested no significant differences under the

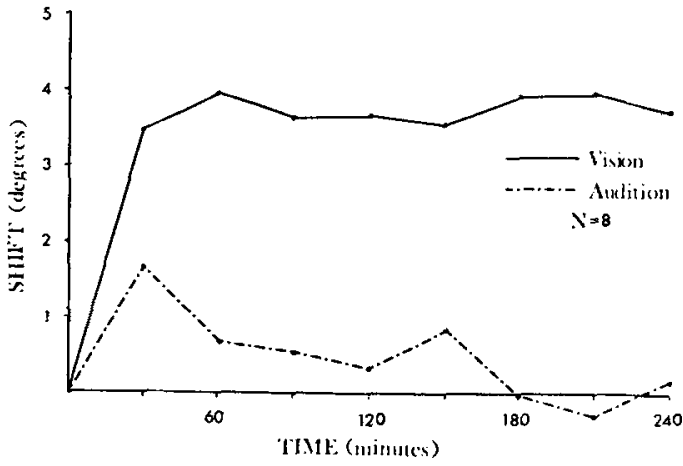

Fig. 2. Mean shifts in visual and auditory median-plane judgments during prolonged exposure to displaced vision: normal auditory stimulation.

control condition. Their mean visual shift was $0.25^{\circ}$ to the left; their mean auditory shift, $0.55^{\circ}$ to the left.

\section{Discussion}

We have found that compensatory shifts in visual median-plane judgments may be accompanied by parallel, maladaptive shifts in auditory median-plane judgments. Why should this occur? It is important to bear in mind that the localization of objects in three-dimensional space is not ordinarily a purely visual-motor task. Other sense modalities are involved although spatial information processed through different modalities is phenomenally congruent. Putting on prism goggles radically disturbs this congruence, and visualspatial information conflicts with veridical information from other modalities. Owing to the customary Importance assigned to vision and the preponderance of visual-spatial information, compensation for the visual transformation may modify some schema common to all spatial senses (Freedman, 1961). This modification initially is accompanied by errors in sensorimotor behavior mediated by nonvisual modalities since they have not been transformed. As visual localization returns to the veridical, auditory and proprioceptive localizations will be in error. To restore accurate functioning, a second change must occur, eliminating the errors in audition and proprioception while maintaining the readjustment of visual-motor behavior. At this point, spatially oriented behavior mediated by both modalities will again be congruent so long as the prism-induced transformation is maintained.

The data shown in Figs. 1 and 2 lend support to this interpretation. With the first 4-h Ss (those wearing muffs), auditory direction-finding did not return to the veridical until after about $180 \mathrm{~min}$. It would appear that initially there was not available to the organism sufficient auditory information to resolve the conflict between transformed visual-spatial information and veridical auditory information. Two plausible reasons may be advanced: (a) the muffs occluded a large portion of the available auditory 
information; and (b) the rate of flow for relevant auditory information is normally much lower than that for visual information. As compensatory shifts in visual midline judgments developed, similar auditory shifts occurred. Relatively long exposures were needed for $S s$ to assimilate sufficient information about auditory errors to correct their auditory direction-finding.

The last group of $\mathrm{Ss}$, who were permitted normal auditory stimulation, apparently received sufficient information about their auditorily guided errors in less than $60 \mathrm{~min}$, and thus were able to resolve the visual-auditory conflict more rapidly.

\section{References}

Craske, B. Intermodal transfer of adaptation to displacement. Nature, 1966, $210,765$.

Freedman, S. J. Perceptual changes in sensory deprivation: suggestions for a conative theory. J. nerv. ment. Dis., 1961, 132, 17-21.

Freedman, S. J., \& Gardos, G. Compensation for auditory re-arrangement and transfer to hand-eye coordination. Paper read at MIT Conference on Adaptation, Cambridge, Mass., June, 1965.
Harris, C. A: Adaptation to displaced vision: visual, motor, or proprioceptive change? Science, 1963, 140, 812-813.

Hay, J. C., \& Pick, H. L., Jr. Visual and proprioceptive adaptation to optical displacement of the visual stimulus. J. exp. Psychol., $1966,71,150-158$.

Smith, K. U., \& Smith, W. M. Perception and motion. Philadelphia: Saunders, 1962.

Wohlwill, J. F. Perceptual learning. Annt. Rev. Psychol., 1966. $17,201-232$.

\section{Hote}

1. From the Neuropsychology Laboratory, Tufts University, Medford, Massachusetts 02155 . The experiments described formed the basis of a doctoral dissertation presented by the senior author to Brandeis University while he was an NIMH Pre-doctoral Fellow (5-F1-MH-25, 670-02) and were presented at a symposium. "The Neuropsychology of Spatial Oriented Behavior " at Tufts University, June 1966. The program of research at the Neuropsychology Laboratory is supported by the Vocational Rehabilitation Administration, Department of Health, Education, and Welfare under Grant No. RD-1890-P and by the Air Force Office of Scientific Research of the Office of Aerospace Research under Contract No. AF49(638)1282.

(Accepted for publication June 19, 1967.) 